\title{
Ein Leben für das Herz
}

\section{Adrian Ritter}

Freier Journalist

Der Kardiologe Thomas Lüscher ist Leiter der Klinik für Kardiologie am UniversitätsSpital Zürich. In seiner Forschung ergründet er die Ursachen der Arteriosklerose und sucht nach neuen Behandlungswegen. Protokoll einer Tagesreise in die Welt des Endothels.

\section{April 2016, 8.15 Uhr: "Campus Schlieren», Universität Zürich}

Wir befinden uns im vierten Stock eines Neubaus im Industriequartier in Schlieren. Wöchentliches «LabMeeting» des Zentrums für molekulare Kardiologie. Das rund 35-köpfige Team besteht zumeist aus jungen Biologinnen und Biologen. Im Mittelpunkt ihrer Forschung steht die Frage: Welche molekularen Mechanismen führen zu Arteriosklerose? Wie lässt sie sich verhindern, wie behandeln? Das Team hat sich im Seminarraum versammelt. Heute stellen eine Doktorandin und ein Masterstudent ihre Forschungsprojekte vor. In der vordersten Reihe sitzt auch Thomas Lüscher, Leiter des Zentrums, fragt nach und gibt Hinweise auf Aspekte, die in der Studie ebenfalls berücksichtigt werden sollten. Thomas Lüscher ist ordent- licher Professor für Kardiologie und kardiovaskuläre Physiologie an der Universität Zürich, Herausgeber des European Heart Journal und der Zeitschrift Cardiovascular Medicine - und einer der meistzitierten Kardiologen weltweit. Kein Wunder, kommen auf eine Doktorandenstelle am Zentrum in Schlieren 220 Bewerbungen, wie es kürzlich der Fall war.

Thomas Lüscher war breit interessiert, als er Anfang der 1970er Jahre in Zürich mit dem Studium begann. Zuerst besuchte er parallel Vorlesungen in Philosophie und Medizin. Wäre Psychiatrie das Richtige für ihn? Oder Neurologie? Der plötzliche Herztod seines Vaters - ein Hausarzt - lenkte das Interesse von Thomas Lüscher definitiv auf die Kardiologie. Nach dem Studium und Tätigkeiten in der Inneren Medizin vertiefte er sich in den 1980er Jahren in den USA in die For-

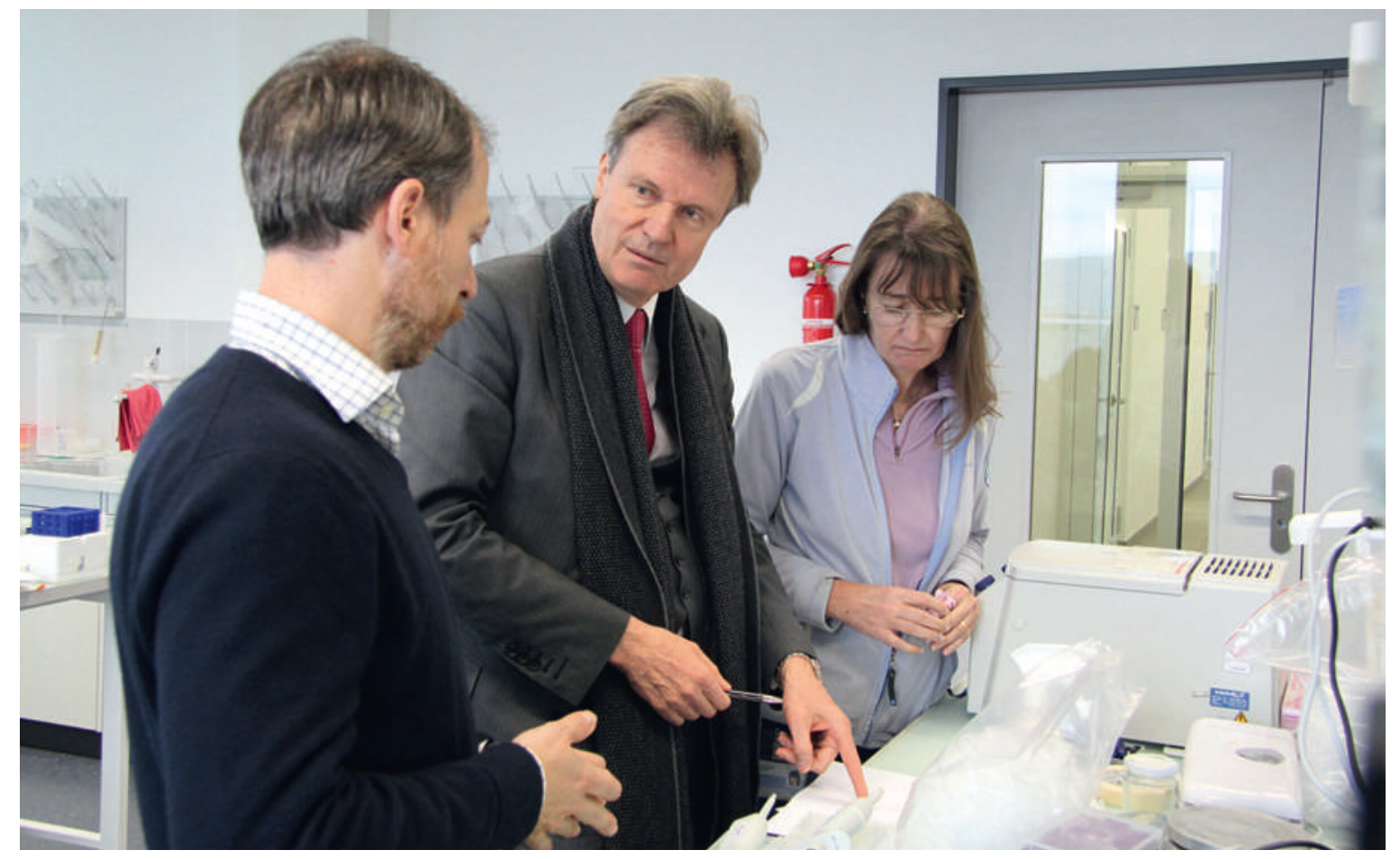

Thomas Lüscher (Mitte) bespricht sich mit zwei Mitgliedern seines Forschungsteams. 


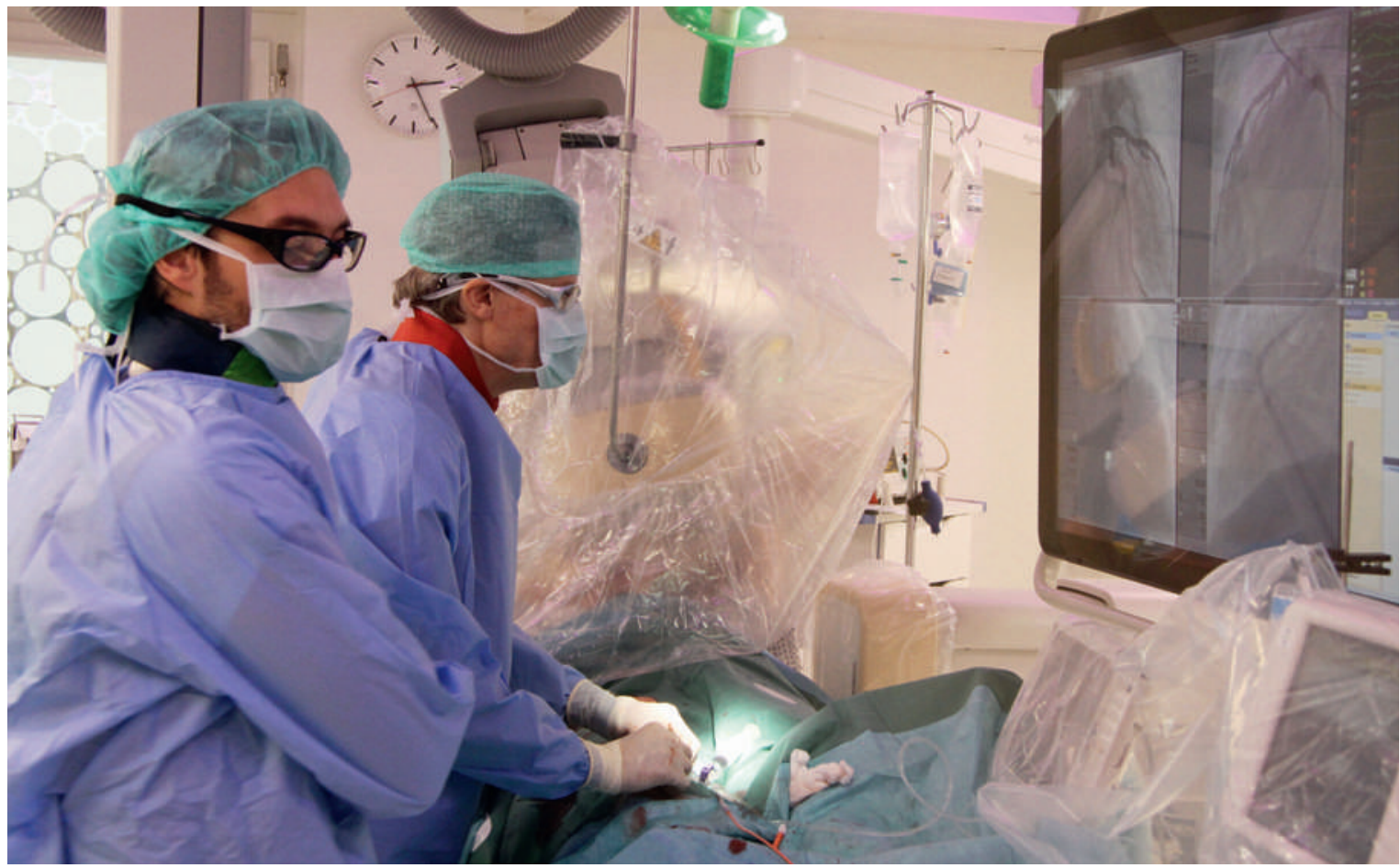

An der Arbeit im Herzkatheterlabor (rechts Thomas Lüscher).

schung über Blutgefässe. Zurück in der Schweiz war er erst an den Universitäten Basel und Bern tätig, ab 1996 dann in Zürich.

Sein Forschungsschwerpunkt ist das Endothel, die innerste Zellschicht der Blutgefässe. Hier richtet die Arteriosklerose ihren Schaden an. Lüscher konnte zeigen, dass dies schon lange vor der Plaque-Bildung beginnt. Hohe LDL-Cholesterinwerte, hoher Blutdruck oder Diabetes verursachen zuerst Funktionsstörungen an den Zellen des Endothels. In der Folge wird Cholesterin eingelagert und Entzündungsreaktionen werden ausgelöst, was die Zellen weiter verändert. Das Resultat: Plaquebildung und ein Verschluss des Blutgefässes. Fatal daran: Auch wenn nur das Endothel verändert ist, vermutet der Körper ein Loch in der Gefässwand und aktiviert die Gerinnung. Mit diesen Erkenntnissen wurde Thomas Lüscher weltweit rund $54000 \mathrm{Mal}$ zitiert. «Wir betreiben Grundlagenforschung mit einem engen Bezug zur Klinik, also translationale Forschung», sagt Lüscher.

\subsection{Uhr: Auf dem Weg von Schlieren ins UniversitätsSpital Zürich}

Unterwegs zu den Auswirkungen von Arteriosklerose - hauptsächlich Angina pectoris, Herzinfarkt und Hirnschlag. Thomas Lüscher ist Direktor der Klinik für Kardiologie, die gemeinsam mit der Klinik für Herzchirurgie das Herzzentrum am UniversitätsSpital Zürich bildet. Während der Autofahrt quer durch die Stadt erkundigt sich eine seiner Sekretärinnen, wann er im Herzkatheterlabor eintreffen wird und ein geplanter Eingriff stattfinden kann. Die Klinik für Kardiologie zählt jährlich rund 3500 stationäre und 10000 ambulante Patienten. Lüscher behandelt täglich ein bis drei Patienten, zumeist Privatversicherte, die bisweilen dafür aus Deutschland, Italien oder Nahost anreisen.

\subsection{Uhr: Stationäre Abteilung der Klinik für Kardiologie}

Thomas Lüscher führt mich durch die Klinik, wird da und dort kurz von einer Assistenzärztin, einem Assistenzarzt um Rat gefragt. Bei der Visite besuchen wir einen jüngeren Patienten, der an einer Herzmuskelerkrankung leidet. Er probiert gerade eine "Life West» an, eine Weste mit eingebautem Defibrillator. Stellt dieser eine Herzrhythmusstörung fest, schlägt er Alarm. Quittiert der Patient diesen nicht, geht das Gerät von einer Bewusstlosigkeit aus und verabreicht einen Schock. Es ist der einzige junge Patient, dem wir an diesem Morgen begegnen. Ansonsten dominiert «70plus». «Der Altersdurchschnitt der von uns behandelten Patienten ist gestiegen", sagt Lüscher. Die Lebenserwartung ist gestiegen, das Alter bringt aber mehr Arteriosklerose mit sich. «Es ist vielleicht DIE Krankheit des Menschen. Schon Pharao Ramses II. litt daran. Wir Menschen haben evolutionär bedingt im Vergleich zu Tieren einen hohen LDL-Cholesterinwert. 
Das mag uns früher gegen Infektionen nützlich gewesen sein. Heute aber schädigt es die Gefässe.» Während der Zeit seines Studiums waren Kardiologen vor allem Diagnostiker, blickt Lüscher zurück. Was man tun konnte: eine Gefässverengung feststellen und den Patienten für einen Bypass in die Herzchirurgie schicken. Das hat sich grundlegend verändert: «Heute machen die Herzchirurgen am USZ rund 380 BypassOperationen, wir Kardiologen rund 1500 koronare Kathetereingriffe jährlich.» Die Kardiologie könne ihre Patienten heute zwar noch immer nicht heilen, aber gut behandeln. Es sei viel erreicht worden in den vergangenen Jahrzehnten: Ballondilatation, mehrere Generationen von Stents, neue Medikamente zur Blutverdünnung und gegen hohen Blutdruck, verbesserte Herzschrittmacher.

12.00 Uhr: Hörsaal des UniversitätsSpitals Zürich Besuch im internationalen Weiterbildungsseminar über Herzversagen. Organisiert wird es von Zurich Heart House, der Stiftung für Herz- und Kreislaufforschung, die Thomas Lüscher 1994 gegründet hat. Ihr Hauptziel: Spenden suchen, um die Forschung voranzubringen. Allein von ehemaligen Patienten sind in den letzten zehn Jahren Donationen in der Höhe von rund 17 Millionen Franken eingegangen. Der ehemalige Emir von Katar hat im vergangenen Sommer 11 Millionen zugesagt, und ermöglicht damit zwei Forschungsprogramme mit vier neuen Assistenzprofessuren.

Thomas Lüscher kann die Aufregung um Drittmittel für die Hochschulen nicht verstehen: «Gelder von Pharmafirmen haben die Unabhängigkeit meiner Forschung noch nie beeinträchtigt. Im Gegenteil: Wer mit den unterschiedlichsten Firmen zusammenarbeitet, kann

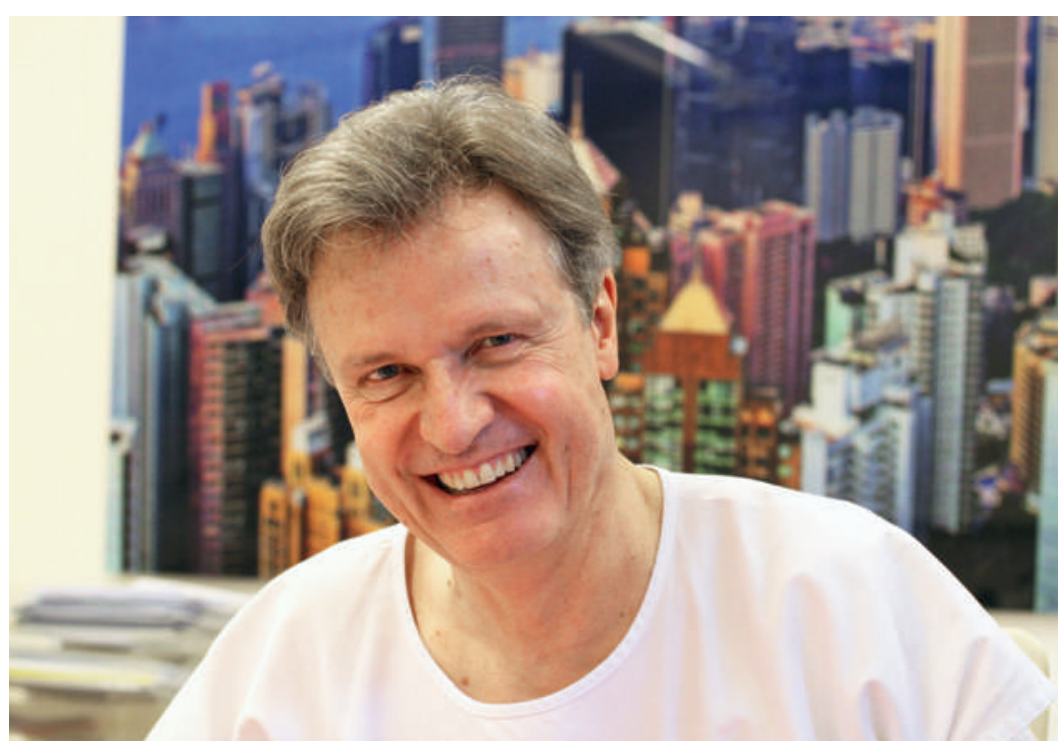

Wirkt auch mit 63 Jahren kein bisschen müde: Thomas Lüscher. bei einem unguten Gefühl auch eher einmal Nein sagen, weil er Alternativen hat.»

\subsection{Uhr: Andreas-Grüntzig-Herzkatheterlabor}

Herr Stucki (Name geändert) liegt in einem der zwei Behandlungsräume des Herzkatheterlabors. Benannt ist es nach dem Kardiologen Andreas Grüntzig, der am UniversitätsSpital Zürich in den 1970er Jahren die Ballondilatation entwickelt hat. Herr Stucki hat das gewünschte Beruhigungsmittel bereits eingenommen. Es ist nicht der erste Herzkathetereingriff für den über 70-jährigen Angina-pectoris-Patienten. Er leidet neuerdings bei Anstrengung vermehrt unter Schmerzen in der Brust.

Das Angiogramm zeigt visuell keine auffälligen Verengungen. Mit einer zusätzlichen Adenosingabe testet Lüscher, in welchem Ausmass sich die betroffene Arterie dehnen lässt. Der Test zeigt doch eine Verengung. Zwei zusätzliche Stents sollen Herrn Stucki in Zukunft wieder freier atmen lassen.

Nach einem schwierigen Eingriff träumt Thomas Lüscher manchmal von Angiogrammen. Etwa vergangene Woche, als ein junger Mann aus Sri Lanka nachts notfallmässig mit einem Infarkt eingeliefert wurde: «Ich musste alle Tricks anwenden, um das verstopfte Blutgefäss zu finden.» Menschen aus Sri Lanka und Indien haben genetisch bedingt schlechtere Blutgefässe, erläutert er.

\subsection{Uhr: Osttrakt UniversitätsSpital Zürich, Büro von Thomas Lüscher}

Er füllt den Bericht über Patient Stucki aus. Die zunehmende Administration. Die Fallpauschalen. Die Ethikkommissionen. Es sind Themen, die Thomas Lüscher immer wieder den Kopf schütteln und ihn zum Essayisten werden lassen - nachzulesen etwa in der kürzlich erschienenen Textsammlung (vgl. Kasten). «Ich kann damit auch meinen Ärger loswerden», sagt er lachend. Am meisten ärgert ihn im Moment die Regulierungswut: «Wir sind eine risikovermeidende Gesellschaft geworden. Je besser es uns geht und je weniger Bedeutung die Religion hat, desto mehr sind wir auf das Diesseits fixiert. Und da darf nichts schiefgehen. Deshalb verhindern wir vieles. Ich bezweifle, dass Andreas Grüntzig für die Entwicklung der Ballondilatation heute noch eine Bewilligung erhalten würde.» Dabei seien Fortschritte in der Forschung dringend nötig, viele Fragen noch ungeklärt. So sind die Forschenden in Schlieren den molekularen Zusammenhängen auf der Spur, warum ein hoher Fleischkonsum zu Arteriosklerose führen kann. Oder welcher $\mathrm{Zu}$ sammenhang zwischen Rheuma und Arteriosklerose besteht. 
Faszinierend findet Thomas Lüscher auch die Forschung zum «Langlebigkeitsgen» SIRT1 und zum Altergen p66 $6^{\text {shc }}$. SIRT1 wird natürlicherweise in der Leber gebildet, lässt den LDL-Cholesterinpegel im Blut sinken und schützt damit die Blutgefässe. Im Alter exprimiert der menschliche Körper weniger SIRT1 und mehr p66 $6^{\text {shc }}$. Dies lässt auch die Blutgefässe altern. Forscher suchen deshalb nach Wegen, den Körper weniger $p 66^{\text {shc }}$ und mehr SIRT1 produzieren zu lassen. Die Vision: Eine Pille, welche die Alterung der Blutgefässe hinausschiebt - auch bei bestehenden Risikofaktoren.

Eine Kapitulation vor dem ungesunden Lebensstil der Menschen? «Ich erzähle allen, sie sollen mit dem Rauchen aufhören, abnehmen und sich mehr bewegen. Aber wir müssen realistisch sein: Nur wenige schaffen es, ihren Lebensstil dauerhaft zu verändern», sagt Lüscher. Ein erstes Medikament war zwar im Tierversuch erfolgreich, schaffte aber wegen Nebenwirkungen beim Menschen die Marktzulassung nicht.

Der Fortschritt ist nicht aufzuhalten, davon ist Lüscher überzeugt. In wenigen Jahren werden sich Menschen einen Chip in die Aorta implantieren lassen können, der permanent ihren Blutdruck misst. Und wenn in fernerer Zukunft im Blut zirkulierende Nanoroboter unsere Gefässe permanent nach Verengungen absuchen? «Das wäre toll», so Lüscher.

\subsection{Uhr: Es klopft an der Bürotüre ...}

Das Redaktionsteam des European Heart Journal steht vor der Türe. Sie treffen sich mehrmals wöchentlich mit Thomas Lüscher, um die nächste Ausgabe zu besprechen.

Lüschers Arbeitstage sind lang, eine 75-Stunden-Woche ist eher die Regel als die Ausnahme. Am Samstag hat er oft Besprechungen mit seinen Forschenden und am Sonntag steht die Lektüre von Manuskripten an. Trotzdem wirkt Thomas Lüscher auch mit 63 Jahren kein bisschen müde. Seine Arbeit scheint auf ihn dieselbe Wirkung zu haben wie ein Langlebigkeitsgen. Der Enthusiasmus, mit dem er darüber berichtet, ist ansteckend.

Adrian Ritter ist freischaffender Journalist. Daneben arbeitet er als Redaktor von UZH News, dem Online-Magazin der Universität Zürich.
Neue Publikationen von Thomas Lüscher

Thomas F. Lüscher / Ai-Phuong Tran / Martin Meyer / Jan Steffel Das gesunde und das kranke Herz

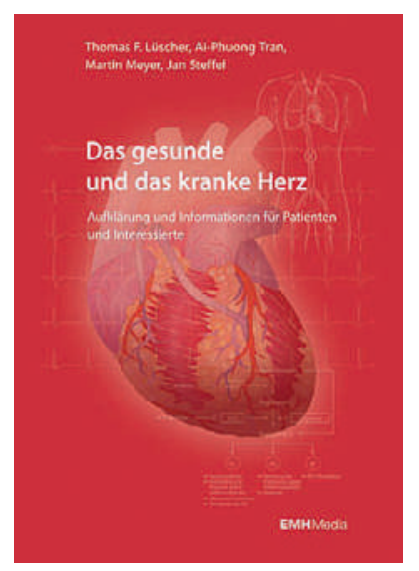

Aufklärung und Information für Patienten und Interessierte Muttenz: EMH Schweizerischer Ärzteverlag, 2015 207 Seiten. 29 CHF. ISBN 978-3-03754-086-2

Thomas F. Lüscher

Über das Herz hinaus

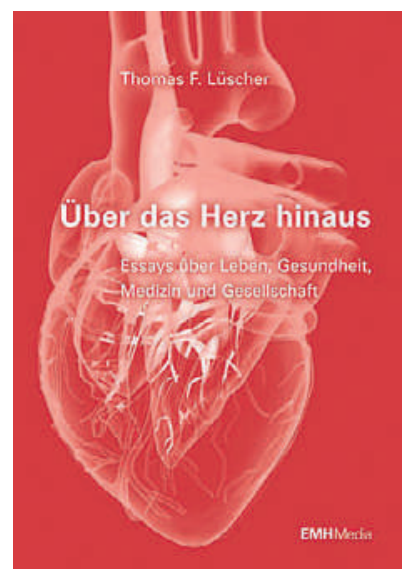

Essays über Leben, Gesundheit, Medizin und Gesellschaft Muttenz: EMH Schweizerischer Ärzteverlag AG, 2015 255 Seiten. 45 CHF. ISBN 978-3-03754-085-5

\section{Bildnachweis}

Fotos: Adrian Ritter. Buchumschläge: EMH. 